\title{
Matrix solution to longitudinal impedance of multi-layer circular structures
}

\author{
H. Hahn
}

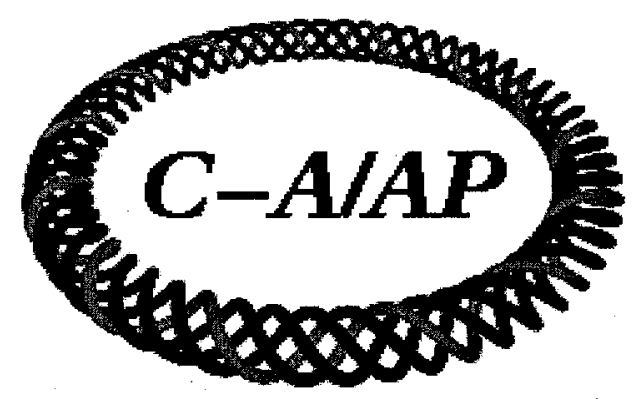

Collider-Accelerator Department Brookhaven National Laboratory Upton, NY 11973

Notice: This document has been authorized by employees of Brookhaven Science Associates, LLC under Contract No. DE-AC02-98CH10886 with the U.S. Department of Energy. The United States Government retains a nonexclusive, paid-up, irrevocable, world-wide license to publish or reproduce the published form of this document, or allow others to do so, for United States Government purposes. 


\title{
Matrix solution to longitudinal impedance of multi-layer circular structures
}

\author{
H. Hahn \\ Brookhaven National Laboratory, Upton, NY 11973-5000
}

A matrix method in which radial wave propagation is treated in analogy to longitudinal transmission lines is presented and applied to finding the longitudinal coupling impedance of axially symmetric multi-layer beam tubes. The method is demonstrated in the case of a Higher Order Mode ferrite absorber with an inserted coated ceramic beam tube. The screening of the ferrite damping properties by the dielectric beam tube is discussed.

PACS numbers: $41.20 . \mathrm{Jb}$

Keywords: Multi-layer beam tube, Higher-order mode ferrite absorber

\section{INTRODUCTION}

The superconducting electron gun for the Energy Recovery Linac (ERL) at the Relativistic Heavy Ion Collider (RHIC) is equipped with a ferrite Higher Order Mode (HOM) absorber [1]. Producing polarized electron beams requires ultimate beam vacuum which is facilitated by separating the ferrite absorber from the gun with an inserted ceramic beam tube. The resulting shielding of the absorber, that is the reduction of the ferrite damping properties, by the ceramic tube represents one disadvantage of this configuration and needs to be studied. The absorber assembly is best analyzed as a longitudinally uniform multi-layer beam tube for which longitudinal coupling impedance expressions can be derived. Zotter [2,3], in a seminal publication presented a solution based on an algorithm involving sequential matching of radial wave impedances. Although this method in principle allows many layers, the numerical implementation becomes increasingly complex and is conveniently applied to a double layer beam tube only. The matrix method presented in this paper is more convenient in solving the fourto-five layer HOM absorber structure.

In the course of the study of the coated ceramic beam tube in the RHIC injection kicker, Hahn noticed the analogy of radial with longitudinal wave transmission and developed the corresponding method in which the sequential wave impedance matching is replaced with multiplication of appropriate matrices relating the electric and magnetic field components in each layer [4]. The sequential matching algorithm and the matrix multiplication are in substance equally valid, but the changes in implementation make the matrix method into a simpler application for multi-layer beam tube structures.

The matrix solution presented here is characterized by a strict separation of the impedance contribution from the space charge and from the surface impedance at the beam tube wall. The beam tube impedance is obtained as eigen solution and is independent of the driving current. Continuity of radial power flow in the absence of the driving current is assured by appropriate constraints on the matrix describing the layer. Although often referred to as field matching the algorithm involves the radial wave impedances at subsequent boundaries whereas the matrix method uses the electric and magnetic field components directly. 
In the present paper, the matrix solution is applied to the ERL electron gun absorber, which is represented by a longitudinally uniform and axial symmetric multi-layer circular structure, both for the coated ceramic beam tube and the ferrite absorber proper. The inner radius of the absorber structure is identified as $b$, the metallic coating extends from $b$ to $r_{1}$, and the ceramic tube from $r_{1}$ to $r_{2}$. An air gap from $r_{2}$ to $r_{W}$ separates the ferrite absorber which then ranges from $r_{W}$ to $r_{o}$. Since of no practical consequence, the absorber is assumed to be enclosed with a perfect conducting infinitely extended metal cylinder. Full matrix expression as well as appropriate approximations or asymptotic expansions are given for all regions. The longitudinal coupling impedance seen by the beam is derived by the matrix method and the shielding of the typical $C 48$ ferrite absorber is found in the important frequency range above $\sim 500 \mathrm{MHz}$ where the tube finite length is of no concern.

\section{MATRIX SOLUTION}

\section{Beam Tube Coupling impedance.}

The electro magnetic field and its associated coupling impedance in a longitudinally uniform axially symmetric circular beam tube excited by a time harmonic current, $i e^{-j k_{z} z} e^{j \omega t}$ are conveniently derived from the wave equation for the longitudinal electric field component, $E_{z}$

$$
\frac{d^{2} E_{z}}{d r^{2}}+\frac{d E_{z}}{r d r}+v^{2} E_{z}=j v^{2} Z_{v} i
$$

In order to prevent a logarithmic divergence of the result for the space charge impedance, the beam can be given a finite transverse size. Solving the case of a tubular beam with radius, $a$, avoids the divergence. The tubular beam of current $I$ is assumed to travel in $z$-direction with velocity $\beta c$ and has the current density

$$
i=\frac{I}{2 \pi a} \delta(r-a) e^{-j k_{z}} e^{j \omega t}
$$

with $k=\omega / c$ and $k_{z}=k / \beta$.

In a circular symmetric geometry, the e.m. monopole fields in any cylinder region

$$
\begin{aligned}
& E_{z}(r)=Z_{v} H_{\Theta}(r) \\
& H_{\Theta}(r)=-j \frac{\varepsilon_{S} k}{v} \frac{d E_{z}(v r)}{d(v r)}=\frac{1}{Z_{v}} \frac{d E_{z}(v r)}{d(v r)}
\end{aligned}
$$

are formed as linear combinations of cylinder function, written in terms of Bessel functions with argument $(v r)$ or in terms of modified Bessel functions with argument $(\kappa r)$. The radial propagation constants, $v^{2}=\left(\mu \varepsilon_{S}-\beta^{-2}\right) k^{2}$ and $\left(-v^{2}\right) \rightarrow \kappa^{2}=\left(\beta^{-2}-\mu \varepsilon_{S}\right) k^{2}$ are determined by the material parameters permeability $\mu / \mu_{0}=\mu^{\prime}-j \mu^{\prime \prime}$ and permittivity plus conductivity $\varepsilon_{S} / \varepsilon_{0}=\varepsilon^{\prime}-j\left(\varepsilon^{\prime \prime}+\sigma / \omega \varepsilon_{0}\right)$.

It is important to note that the total coupling impedance consists of two parts, the energy dependent space charge plus a separate contribution from the beam tube as 
discussed in the Appendix. The coupling impedance of a filamentary beam in the relativistic limit follows as

$$
\mathbb{Z} \approx-\frac{\kappa Z_{C}}{2 \pi} \ln \left(\frac{b}{a}\right)-\frac{Z(b)}{2 \pi b}\left(\frac{2}{2-\kappa b Z(b)}\right)
$$

with $\kappa=k / \beta \gamma, Z_{C}=-j Z_{0} / \beta \gamma$, and $\gamma=1 / \sqrt{1-\beta^{2}}$. In the extreme relativistic case, $\kappa \rightarrow 0$, usually assumed for the coupling impedance calculations, one finds [5]

$$
\mathbb{Z} \approx-\frac{Z(b)}{2 \pi b}
$$

with $Z(b)$ the surface impedance or radial wave impedance at the beam tube wall, which suggests to ignore the space charge impedance and for the present task to focus on the impedance from the eigen solution for the absorber structure tube alone.

\section{Radial Wave Matrices}

The coupling impedance of a multi-layer structure is obtained by properly matching of the tangential field components at the cylinder boundaries which in full analogy to the treatment of longitudinal transmission lines is best done with radial transfer matrices. The matrix for a layer, ranging from the inner radius $r_{i}$ to the outer radius $r_{o}$ is given in the general form (and in natural units to stream line the notation, but $Z_{0} \approx 377$ if explicitly shown)

$$
\left(\begin{array}{c}
E_{z}\left(r_{i}\right) \\
H_{\Theta}\left(r_{i}\right)
\end{array}\right)=M=\left[\begin{array}{ll}
m_{e e} & m_{e h} \\
m_{h e} & m_{h h}
\end{array}\right]\left(\begin{array}{c}
E_{z}\left(r_{o}\right) \\
H_{\Theta}\left(r_{o}\right)
\end{array}\right)
$$

with the elements satisfying certain constraints to achieve power flow in addition to field component matching. At the outer radius, $r=r_{o}$, it is required that the matrix reads as

$$
M\left(r=r_{o}\right)=\left[\begin{array}{ll}
1 & 0 \\
0 & 1
\end{array}\right]
$$

In full analogy to axial transmission line theory, the matrix of a sequence of radially spaced cylinders is found as the sequential product of all individual matrices, starting from the most inward $M^{I}$

$$
M=M^{I} M^{I I} M^{I I I} \ldots
$$

In contrast to transmission line theory which relates voltages and currents, the matrix here relates electric and magnet fields imposing the constraint of power flow equality by the requirement (at all $\left.r_{i}<r<r_{o}\right)$ that

$$
\operatorname{det} M=r_{o} / r \text {. }
$$

Rigorous expressions for the matrix elements are given in terms of Bessel functions or modified Bessel functions and the choice depends only on which asymptotic expansion is better suited to a particular material parameter range. The ceramic beam tube is described with Bessel functions of order zero and argument, $(v r)$ 


$$
\begin{aligned}
& m_{e e}=\frac{\pi v r_{o}}{2}\left[Y_{0}(v r) J_{1}\left(v r_{o}\right)-J_{0}(v r) Y_{1}\left(v r_{o}\right)\right] \\
& m_{h e}=j \frac{\pi \varepsilon_{S} k r_{o}}{2}\left[Y_{1}(v r) J_{1}\left(v r_{o}\right)-J_{1}(v r) Y_{1}\left(v r_{o}\right)\right] \\
& m_{e h}=-j \frac{\pi v^{2} r_{o}}{2 \varepsilon_{S} k}\left[Y_{0}\left(v r_{o}\right) J_{0}(v r)-J_{0}\left(v r_{o}\right) Y_{0}(v r)\right] \\
& m_{h h}=\frac{\pi v r_{o}}{2}\left[Y_{0}\left(v r_{o}\right) J_{1}(v r)-J_{0}\left(v r_{o}\right) Y_{1}(v r)\right]
\end{aligned}
$$

Broad coverage for other materials is found with modified Bessel functions of order zero and argument $(\kappa r)$

$$
\begin{aligned}
& m_{e e}=\kappa r_{o}\left[K_{0}(\kappa r) I_{1}\left(\kappa r_{o}\right)+I_{0}(\kappa r) K_{1}\left(\kappa r_{o}\right)\right] \\
& m_{h e}=-j \varepsilon_{S} k r_{o}\left[K_{1}(\kappa r) I_{1}\left(\kappa r_{o}\right)-I_{1}(\kappa r) K_{1}\left(\kappa r_{o}\right)\right] \\
& m_{e h}=j \frac{\kappa^{2} r_{o}}{\varepsilon_{S} k}\left[K_{0}(\kappa r) I_{0}\left(\kappa r_{o}\right)-I_{0}(\kappa r) K_{0}\left(\kappa r_{o}\right)\right] \\
& m_{h h}=\kappa r_{o}\left[K_{1}(\kappa r) I_{0}\left(\kappa r_{o}\right)+I_{1}(\kappa r) K_{0}\left(\kappa r_{o}\right)\right]
\end{aligned}
$$

with the radial propagation constant in each layer determined by the material parameters given above. The wave impedances are for the ceramic tube $Z_{v}=j v /\left(\varepsilon_{S} k\right)$ and for the other materials $Z_{\kappa}=-j \kappa /\left(\varepsilon_{S} k\right)$.

In high-conductivity metals $\varepsilon_{S} \approx-j \sigma / k$ leading to $\kappa \rightarrow \chi=\sqrt{j \mu \sigma k}$, and $Z_{\kappa} \rightarrow Z_{z}=\sqrt{j \mu k / \sigma}=(1+j) \sqrt{\mu k / \sigma}$, suggesting the use of Hankel-functions instead, which however are not supported by the Mathematica program and thus are not used here.

The coupling impedance seen by the beam follows according Equ. 5 from the surface impedance $Z\left(r_{i}\right)$ at the inner beam tube radius, which in turn is determined by the wave impedance, $Z\left(r_{o}\right)$, at the outer radius of the beam tube

$$
\begin{aligned}
& Z\left(r_{i}\right)=\frac{E_{z}\left(r_{o}\right) m_{e e}+H_{\Theta}\left(r_{o}\right) m_{e h}}{E_{z}\left(r_{o}\right) m_{h e}+H_{\Theta}\left(r_{o}\right) m_{h h}} \\
& =\frac{Z\left(r_{o}\right) m_{e e}+m_{e h}}{Z\left(r_{o}\right) m_{h e}+m_{h h}}
\end{aligned}
$$

This procedure can be generalized by pointing out that the defining impedance at the innermost radius is determined via the overall matrix by the wave impedance of the outermost layer at $r_{o}$. For the specific example of the absorber structure, the inner radius at the coating is $r_{i}=b$ and the outer radius is taken at $r_{W}$ where the surface impedance of the ferrite ring is known.

Although strictly speaking, the outermost layer must be air, in practical terms one can take a perfect conductor, a perfect magnet, a finite thick metal cylinder, or vacuum in the relativistic limit as termination for which the wave impedances are $Z\left(r_{o}\right)=\infty, Z\left(r_{o}\right)=0$, 
$Z\left(r_{o}\right)=\sqrt{j \mu k / \sigma}$, and $Z\left(r_{o}\right)=j \beta \gamma$ respectively. Note that the matrix solution covers these and more general situations equally well without change to the matrices.

\section{LAYERS in the ABSORBER}

In preparation of computing the shielding of the ferrite absorber by the coated ceramic tube, the matrix expressions have been tested separately for the use in the Mathematica program. Full matrix expressions as well as appropriate approximations are here given for all regions. The inner radius of the absorber structure is identified as $b$, the coating extends from $b$ to $r_{1}$, and the ceramic tube from $r_{1}$ to $r_{2}$. An air gap from $r_{2}$ to $r_{W}$ separates the absorber, which then ranges from $r_{W}$ to $r_{o}$. Since of no practical consequence, the ferrite absorber is assumed to be enclosed with a perfect conducting infinitely extended metal cylinder.

\section{Metallic Layer}

The ceramic tube must have an internal metal coating to prevent static charge accumulation, but its thickness is limited by the consequent shielding of the ferrite absorber strength. Following the DESY practice [6] and also used for PETRA III [7], the coating uses Titanium-Stabilized High Gradient Steel (TSHGS) (316Ti in the USA) with $\sigma \approx 2 \times 10^{6} / \Omega \mathrm{m}\left(7.5 \times 10^{8} / \mathrm{m}\right.$ in natural units), $\varepsilon=1, \mu=1$.

The general expression for the radial wave impedance $Z$ of a free-standing metal tube is written in the relativistic limit as

$$
Z(b)=\frac{m_{e e}}{m_{h e}}=-\frac{\chi}{\sigma} \frac{\left[K_{0}(\chi b) I_{1}\left(\chi r_{1}\right)+I_{0}(\chi b) K_{1}\left(\chi r_{1}\right)\right]}{\left[K_{1}(\chi b) I_{1}\left(\chi r_{1}\right)-I_{1}(\chi b) K_{1}\left(\chi r_{1}\right)\right]}
$$

Using the matrix for the thin-layer approximation

$$
\begin{aligned}
& m_{e e} \approx 1+j \frac{1}{2} \sigma k \Delta^{2}\left(1+\frac{1}{3} \frac{\Delta}{b}\right) \\
& m_{h e} \approx-\sigma \Delta\left(1+\frac{1}{2} \frac{\Delta}{b}\right) \\
& m_{e h} \approx-j k \Delta\left(1+\frac{1}{2} \frac{\Delta}{b}\right) \\
& m_{h h} \approx 1+\frac{\Delta}{b}+j \frac{1}{2} \sigma k \Delta^{2}\left(1+\frac{1}{3} \frac{\Delta}{b}\right)
\end{aligned}
$$

one finds

$$
Z(b)=\frac{m_{e e}}{m_{h e}}=-\frac{1+j \sigma k \Delta^{2}(1+\Delta /(3 b)) / 2}{\sigma \Delta(1+\Delta /(2 b))}
$$

In the further simplified long-wavelength limit the beam impedance $\mathbb{Z}=-Z /(2 \pi b)$ follows as

$$
\mathbb{Z}(b) \approx 1 /(2 \pi b \sigma \Delta)
$$

which represents the DC resistance per unit length of the metal cylinder. For example, a $1 \mu \mathrm{m}$ thick TSHGC tube with $5 \mathrm{~cm}$ inner radius has a DC resistance of $\sim 1.6 \Omega / \mathrm{m}$. 
The coupling impedance of a free-standing steel tube, air (vacuum) outside with inner radius $b=5 \mathrm{~cm}$ is shown in Fig. 1, computed for several coating thickness, $\Delta=r_{1}-b$, first with the full matrix given by Equ.13 and then with the "thin layer" approximation. For thin layers, as long as the thickness is smaller than the classical skin depth, $\Delta \leq \delta=\sqrt{2 / \mu \sigma k}$, the Bessel functions and the thin-layer approximation are identical. Reducing the coupling impedance by increasing the thickness is limited at higher frequencies by the skin effect.

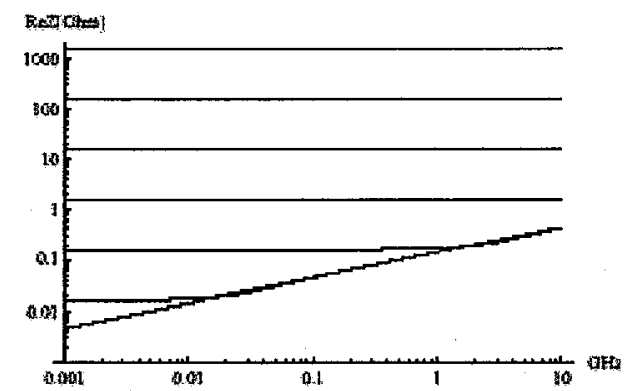

Figure 1. Coupling Impedance of a metal beam tube with various thickness, from $1 \mathrm{~nm}$ (green), $1 \mu \mathrm{m}$ (red) to $1 \mathrm{~mm}$ (blue)

\section{Ceramic Beam Tube}

The case of a ceramic beam tube is of great practical importance and has been addressed by several workers stressing different specific aspects of the solution [8-10]. The ceramic is fully described by a matrix with the radial propagation constants

$v=k \sqrt{\mu \varepsilon-\beta^{-2}}$ where $\mu=1$ and $\varepsilon=\varepsilon^{\prime}(1-j \tan \delta) \approx 9.1(1-j 0.0007)$ for an aluminum oxide. Consistent with its treatment in the literature, only the relativistic approximation will be considered. In the literature two different approximations for the outside region have been discussed, although their relevance to actual structures is not obvious. Air (vacuum) and perfect magnet have been applied for kickers, and perfect conductivity metal for general non-specified structures, which are defined by the wave impedances $Z\left(r_{o}\right)=\infty$ and $Z\left(r_{o}\right)=0$, respectively.

The typical ceramic tube is used with some form of external metallic by-pass leading to the full expression for the radial wave impedance

$$
Z\left(r_{1}\right)=\frac{m_{e h}}{m_{h h}}=-j \frac{v}{\varepsilon_{S} k} \frac{\left[Y_{0}\left(v r_{2}\right) J_{0}\left(v r_{1}\right)-J_{0}\left(v r_{2}\right) Y_{0}\left(v r_{1}\right)\right]}{\left[Y_{0}\left(v r_{2}\right) J_{1}\left(v r_{1}\right)-J_{0}\left(v r_{2}\right) Y_{1}\left(v r_{1}\right)\right]}
$$

For certain purposes, the matrix for the ceramic tube can be represented by the long wavelength limit,

$$
\begin{aligned}
& m_{e e}=1+\frac{1}{4}\left(v r_{1}\right)^{2}\left(1-\frac{r_{2}^{2}}{r_{1}^{2}}+2 \frac{r_{2}^{2}}{r_{1}^{2}} \ln \frac{r_{2}}{r_{1}}\right) \\
& m_{h e}=j \frac{\varepsilon k r_{1}}{2}\left(1-\frac{r_{2}^{2}}{r_{1}^{2}}\right)+j \frac{\varepsilon k v^{2} r_{1}^{3}}{16}\left(1-\frac{r_{2}^{4}}{r_{1}^{4}}+4 \frac{r_{2}^{2}}{r_{1}^{2}} \ln \frac{r_{2}}{r_{1}}\right)
\end{aligned}
$$




$$
\begin{aligned}
& m_{e h}=-j \frac{v^{2} r_{2}}{\varepsilon k} \ln \frac{r_{2}}{r_{1}} \approx-j k r_{2} \sqrt{1-\frac{1}{\varepsilon}} \ln \frac{r_{2}}{r_{1}} \\
& m_{h h} \approx \frac{r_{2}}{r_{1}}\left[1-\frac{1}{4}\left(v r_{1}\right)^{2}\left(1-\frac{r_{2}^{2}}{r_{1}^{2}}+2 \frac{r_{2}^{2}}{r_{1}^{2}} \ln \frac{r_{2}}{r_{1}}\right)\right]
\end{aligned}
$$

from which the known result for the longitudinal impedance per unit length of the uncoated ceramic tube with perfect external short follows (note complex $\varepsilon$ ), [2]

$$
\mathbb{Z}\left(r_{i}\right) \approx \frac{Z_{0} k}{2 \pi} \sqrt{1-\frac{1}{\varepsilon}} \ln \frac{r_{2}}{r_{1}} .
$$

Numerical results for the exact and long-wavelength approximation are readily obtained via the Mathematica program and are shown in Fig. 2 for a beam tube with inner radius $r_{1}=5 \mathrm{~cm}$ and wall thickness of $0.6 \mathrm{~cm}$. Shown is the result from above Equ. 19 (black), the long-wavelength approximation (red) and the exact expressions Equ. 17 (green).

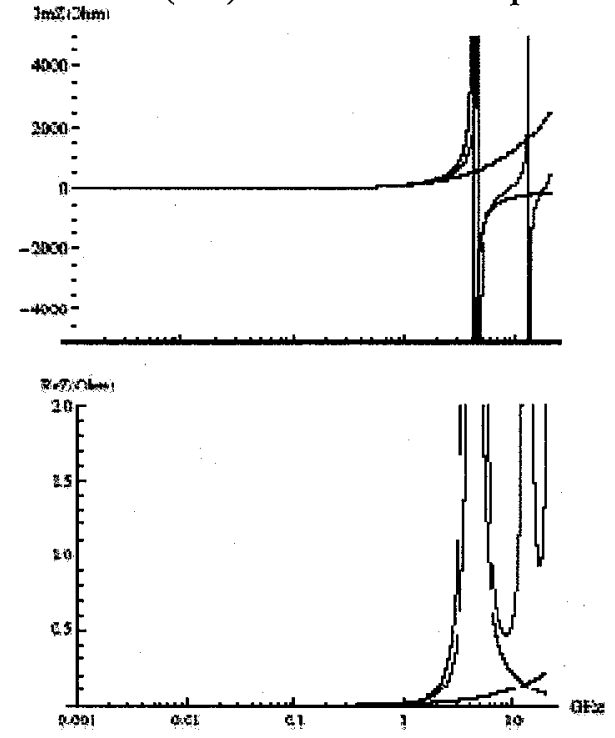

Figure 2. Real and imaginary longitudinal impedance of ceramic beam tube with perfect external conductor from approximate equation (black), the long-wavelength approximation (red) and the exact expressions (green).

\section{Ceramic beam tube with inner coating}

The properties of a ceramic beam tube in the HOM absorber and the screening by an inner metallic coating are studied by treating the external region as infinitely extending vacuum, that is $Z\left(r_{2}\right)=\infty$. The effect of the coating thickness on the coupling impedance is seen in Fig. 3, where the thickness is increased from none (green) to $1 \mathrm{~nm}$ (blue) and 1 $\mu \mathrm{m}$ (red) with two steps in between. The real impedance value is reduced by coating at low frequencies to the DC value of the coating, but it remains increased at higher frequencies. Below the resonance region the coupling impedance can be interpreted as a series RC circuit driven by the beam current. The results point to the need for a careful study of the ceramic coating in the HOM ferrite absorber. 


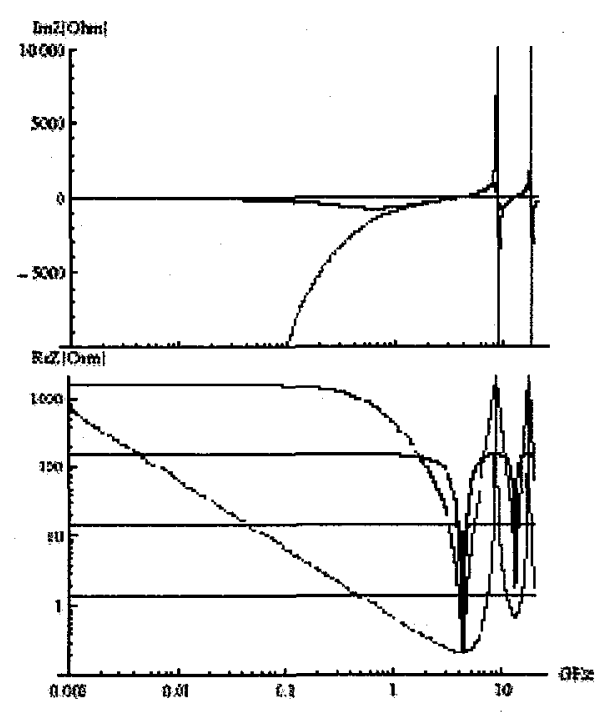

Figure 3. Real and imaginary coupling impedance of a ceramic beam tube for different coating thickness, from none (green), $1 \mathrm{~nm}$ (blue), to $1 \mu \mathrm{m}$ (red)

\section{IV. e-GUN HOM FERRITE ABSORBER}

The HOM ferrite absorber for the superconducting e-gun is assembled from two separate elements. A ceramic beam tube is permanently attached to the gun and serves as break in the vacuum tube, and the ferrite structure is added around it in air. In order to analyze the combination, the matrices for the air space and the ferrite must first be established. The damping properties of the absorber in the absence of the ceramic tube can then be found and the shielding effect is obtained by the comparison with the fully assembled structure.

\section{Matrix of air (vacuum) between two layers}

Following the usual practice, and for the sake of simplicity the small, non-zero electric susceptibility, $\left(\varepsilon^{\prime}-1\right) \approx 6 \times 10^{-4}$, of air is ignored and treated as vacuum. The exact matrix for vacuum between two layers between, $r_{2}$ and $r_{W}$, follows as

$$
\begin{aligned}
& m_{e e} \simeq 1+\frac{r_{W}^{2} k^{2}}{4(\beta \gamma)^{2}}\left(\frac{r_{2}^{2}}{r_{W}^{2}}-1+2 \ln \frac{r_{W}}{r_{2}}\right) \rightarrow 1 \\
& m_{e h} \simeq j \frac{k r_{W}}{\beta \gamma} \ln \frac{r_{W}}{r_{2}} \rightarrow 0 \\
& m_{h e}=j \frac{k r_{2}}{2 \beta \gamma}\left[1-\left(\frac{r_{W}}{r_{2}}\right)^{2}\right] \rightarrow 0 \\
& m_{h h} \simeq \frac{r_{W}}{r_{2}}\left\{1-\frac{r_{2}^{2} k^{2}}{4(\beta \gamma)^{2}}\left(\frac{r_{W}^{2}}{r_{2}^{2}}-1+2 \ln \frac{r_{W}}{r_{2}}\right)\right\} \rightarrow \frac{r_{W}}{r_{2}}
\end{aligned}
$$

The further simplification for the extreme relativistic limit, used here, is indicated by $\kappa \simeq k / \beta \gamma \rightarrow 0$. 


\section{Ferrite Absorber}

The ferrite absorber is simulated as a longitudinally uniform cylinder, $3 \mathrm{~mm}$ wall thickness, with inner radius $r_{W}=6 \mathrm{~cm}$, bringing the outer structure radius to $r_{o}=6.3 \mathrm{~cm}$. The ferrite is enclosed in a high conductivity metal cylinder, which in view of the broad approximation for the absorber can also be assumed as perfect conducting. The fields in the ferrite are given by

$$
\begin{aligned}
& E_{z}=m_{e h}=j \frac{\zeta^{2} r_{o}}{\varepsilon k}\left[K_{0}\left(\zeta r_{W}\right) I_{0}\left(\zeta r_{o}\right)-I_{0}\left(\zeta r_{W}\right) K_{0}\left(\zeta r_{o}\right)\right] \\
& H_{\Theta}=m_{h h}=\zeta r_{o}\left[K_{1}\left(\zeta r_{W}\right) I_{0}\left(\zeta r_{o}\right)+I_{1}\left(\zeta r_{W}\right) K_{0}\left(\zeta r_{o}\right)\right]
\end{aligned}
$$

with the radial wave number, $\zeta^{2}=(1-\varepsilon \mu) k^{2}$ and the relative material parameters for the C48 ferrite $\mu=65 /\left(1+j 5.5 f_{G H z}\right)$ and $\varepsilon \approx 13$.[11] The radial wave impedance at the ferrite absorber inner radius follows as

$$
Z_{F e}=j \frac{\zeta}{\varepsilon k} \frac{K_{0}\left(\zeta r_{W}\right) I_{0}\left(\zeta r_{o}\right)-I_{0}\left(\zeta r_{W}\right) K_{0}\left(\zeta r_{o}\right)}{K_{0}\left(\zeta r_{W}\right) I_{1}\left(\zeta r_{o}\right)+I_{0}\left(\zeta r_{W}\right) K_{1}\left(\zeta r_{o}\right)}
$$

The resulting coupling impedance in the absence of the ceramic beam tube is shown for the real and imaginary components in Fig. 4.

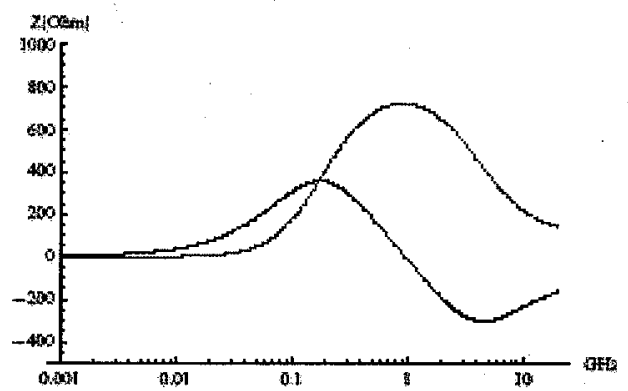

Figure 4: Coupling impedance of free-standing ferrite layer, real (green) and imaginary (red)

\section{Ferrite Absorber with coated ceramic tube}

All required matrix expression for the layers in the assembled ferrite absorber have been developed, and the radial wave impedance at the inner radius can now be written in terms of the overall matrix as

$$
Z(b)=\frac{M_{e e} Z_{F e}+M_{e h}}{M_{h e} Z_{F e}+M_{h h}}
$$

The matrix elements have been obtain by matrix multiplications and read as follows

$$
\begin{aligned}
& M_{e e}=m_{e e}^{\zeta} m_{e e}^{v}+m_{e h}^{\zeta} m_{h e}^{v} \\
& M_{h e}=m_{h e}^{\zeta} m_{e e}^{v}+m_{h h}^{\zeta} m_{h e}^{v} \\
& M_{e h}=\left(m_{e e}^{\zeta} m_{e h}^{v}+m_{e h}^{\zeta} m_{h h}^{v}\right) r_{W} / r_{2} \\
& M_{h h}=\left(m_{h e}^{\zeta} m_{e h}^{v}+m_{h h}^{\zeta} m_{h h}^{v}\right) r_{W} / r_{2}
\end{aligned}
$$

The coupling impedance of the entire structure of HOM ferrite absorber with coated beam tube 


$$
\mathbb{Z}(b)=-Z_{0} \frac{Z(b)}{2 \pi b}
$$

was computed via the Mathematica program and the results are shown in Fig. 5. The impedance was determined for a TSHGS coating thickness of $\Delta=0$ (green), $1 \mathrm{~nm}$ and $1.6 \mathrm{k} \Omega / \mathrm{m}$ (red), and $10 \mathrm{~nm}$ (blue). It is to be noted that even a few Angstrom thick layer on the ceramic tube reduces the effectiveness of the absorber. For reference, the impedance of the freestanding ferrite absorber is also shown in black. The shielding, that is the reduction of the HOM damping by the ferrite becomes at higher frequencies quite pronounced. In fact the only damping is obtained from the dielectric losses of the ceramic. The combination of ferrite with a lossy ceramic beam tube may have to be considered. [12]

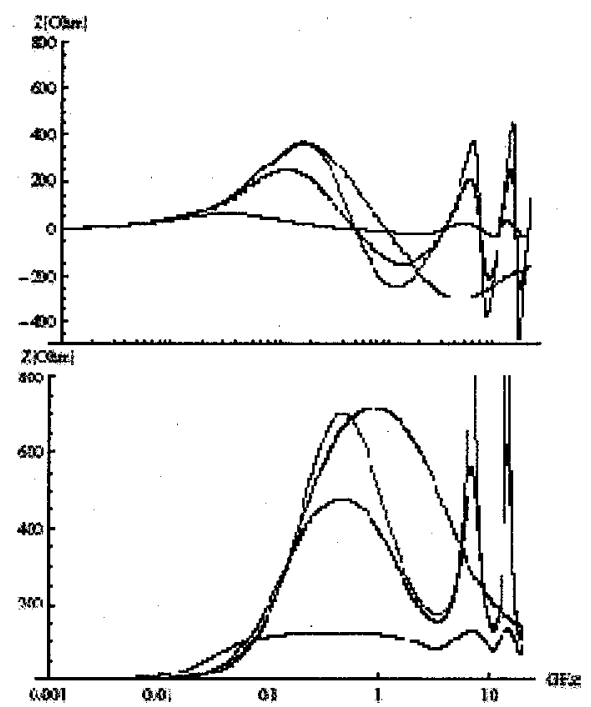

Figure 5: Coupling impedance of HOM ferrite absorber with coated ceramic beam tube. Freestanding ferrite (black), coating thickness none (green $0,1 \mathrm{~nm}$ (red), $10 \mathrm{~nm}$ (blue).

\section{Acknowledgements}

The particular configuration of the HOM absorber for the e-gun was conceived in discussions with Drs. Litvinenko and Ben-Zvi.

\section{APPENDIX}

The foundation for the present study is laid by first deriving the expression for the longitudinal coupling impedance seen by an axial beam in a beam tube with known radial wave impedance. It is important to note that the total coupling impedance of two parts, the energy dependent space charge plus a separate contribution from the beam tube. The beam tube properties are fully defined at the inner tube radius by its radial wave impedance which can be found independently of the beam even in the case of a layered tube (of course assuming only linear materials).

In order to prevent a logarithmic divergence of the result for the space charge impedance, the beam must be given a finite transverse size. Solving the case of a tubular 
beam with radius $a$ avoids the divergence. The tubular beam of current, $I$, is assumed to travel in $z$-direction with velocity $\beta c$ and has the current density

$$
i=\frac{I}{2 \pi a} \delta(r-a) e^{-j k_{z}} e^{j \omega t}
$$

The e.m. fields induced by the tubular beam are solutions of the wave equation in the form of modified Bessel functions with argument $(\kappa r)$. The e.m. fields inside and outside the tubular current are defined by matching the electric field across the tubular current while imposing Amperes law at $r=a$, and satisfying the boundary condition at the beam tube inner radius $E_{z} / H_{\Theta}=Z(b)$. Omitting the time harmonic factor $e^{j\left(k \beta^{-1} z-k t\right)}$ and noting that in contrast to Zotter's papers $k=\omega / c$ one finds the field expressions inside the current tube

$$
\begin{aligned}
& E_{z i}(\kappa r)=G I_{0}(\kappa r) ; \\
& H_{\Theta i}(\kappa r)=G Z_{C}^{-1} I_{1}(\kappa r)
\end{aligned}
$$

and outside of the current

$$
\begin{aligned}
& E_{z o}(\kappa r)=G \frac{I_{0}(\kappa a)\left[K_{0}(\kappa r)+g I_{0}(\kappa r)\right]}{K_{0}(\kappa a)+g I_{0}(\kappa a)}, \\
& H_{\circledast o}(\kappa r)=G Z_{C}^{-1} \frac{I_{0}(\kappa a)\left[-K_{1}(\kappa r)+g I_{1}(\kappa r)\right]}{K_{0}(\kappa a)+g I_{0}(\kappa a)}
\end{aligned}
$$

with $\kappa=k / \beta \gamma$ and $Z_{C}=-j Z_{0} / \beta \gamma$.

Imposing the value of the radial wave impedance and Ampere's law at the beam leads to

$$
g=\frac{Z(b) K_{1}(\kappa b)+Z_{C} K_{0}(\kappa b)}{Z(b) I_{1}(\kappa b)-Z_{C} I_{0}(\kappa b)}
$$

and $\quad G=Z_{C} \frac{I}{2 \pi a} /\left\{\frac{I_{0}(\kappa a)\left[-K_{1}(\kappa a)+g I_{1}(\kappa a)\right]}{K_{0}(\kappa a)+g I_{0}(\kappa a)}-I_{1}(\kappa a)\right\}$

The longitudinal coupling impedance is

$$
\begin{aligned}
& \mathbb{Z}=-\frac{E_{z}(a)}{I}=-Z_{C} \frac{I_{0}(\kappa a)}{2 \pi a} /\left\{\frac{I_{0}(\kappa a)\left[-K_{1}(\kappa a)+g I_{1}(\kappa a)\right]}{K_{0}(\kappa a)+g I_{0}(\kappa a)}-I_{1}(\kappa a)\right\} \\
& =-Z_{C} \frac{\kappa I_{0}(\kappa a)}{2 \pi I_{0}(\kappa b)}\left[I_{0}(\kappa a) K_{0}(\kappa b)-K_{0}(\kappa a) I_{0}(\kappa b)\right]+ \\
& +\frac{Z(b)}{2 \pi b} \frac{I_{0}^{2}(\kappa a)}{I_{0}^{2}(\kappa b)-Z(b) I_{0}(\kappa b) I_{1}(\kappa b)}
\end{aligned}
$$

Considering a filamentary beam, $a \rightarrow 0$, and the relativistic limit $\kappa \approx \frac{k}{\beta \gamma}$ yields

$$
\mathbb{Z}(\kappa \rightarrow 0) \approx \frac{\kappa Z_{C}}{2 \pi} \ln \left(\frac{b}{a}\right)+\frac{Z(b)}{2 \pi b}\left(\frac{2}{2-\kappa b Z(b)}\right)
$$

which is a more general solution to Chao's "Exercise 2.1" but reduces in the extreme relativistic limit to his equation (2.17) [5] 


$$
\mathbb{Z}(\kappa \rightarrow 0) \approx-\frac{Z(b)}{2 \pi b}
$$

Note that the beam tube contribution is fully independent of the current's radial distribution only in the extreme relativistic approximation.

\section{References}

1. I. Ben-Zvi, A. Burrill, R. Calaga, X. Chang, R. Grover, R. Gupta, H. Hahn, L. Hammons, D. Kayran, J. Kewisch, R. Lambiase, V. N. Litvinenko, G.McIntyre, D. Naik, D. Pate, D. Phillips, E. Pozdeyev, T. Rao, J. Smedley, R. Than, R. J. Todd, D. Weiss, Q. Wu, A. Zaltsman, M. Cole, M. Falletta, D. Holmes, J. Rathke, T. Schultheiss, R. Wong, A. Murray, and M. Todd , Proceedings of FEL 2007, Novosibirsk, Russia, p. 290.

2. B. Zotter, Particle Accelerators, 1. p. 311 (1970)

3. B. W. Zotter and S. A. Kheifets, Impedances and Wakes in High-Energy Accelerators, p. 157 (World Scientific Publishing Co., Singapore, 1997)

4. H. Hahn and A. Ratti, The Coupling Impedance of the RHIC Injection Kicker System, RHIC Project Report AD/RHIC/RD-106 (1996)

5. A. W. Chao, Physics of Collective Beam Instabilities in High Energy Accelerators, equ. 2.17, p 45 (John Wiley \& Sons, New York, 1993)

6. M. Schroeder, marco.schroeder@desy.de (private communication)

7. M. Ivanyan, E. Laziev, V. Tsakanov, A. Vardanyan, A. Tsakanian, and R. Wanzenberg, ICFA Beam Dynamics Newsletter 45, p.125 (April 2008)

8. A. Piwinski, PAC 1977, Chicago, IL, p.1364

9. S. S. Kurennoy, PAC 1993, Washington, DC p. 3420

10. M. Ivanyan and V. Tsakanov, Phys. Rev. Special Topics AB, vol 7, 114402 (2004)

11. H. Hahn, E.M. Choi, and L. Hammons, C-AD Report C-A/AP/329 (2008)

12. V. Shemelin, M. Liepe, and H. Padamsee, Nucl.Instr. Meths Phys. Res. A557, p.268 (2006) 